\title{
Feasibility of laparoscopy-assisted total gastrectomy in patients with clinical stage I gastric cancer
}

\author{
Noriko Wada $\cdot$ Yukinori Kurokawa $\cdot$ Shuji Takiguchi $\cdot$ \\ Tsuyoshi Takahashi $\cdot$ Makoto Yamasaki $\cdot$ Hiroshi Miyata \\ Kiyokazu Nakajima $\cdot$ Masaki Mori $\cdot$ Yuichiro Doki
}

Received: 25 October 2012 / Accepted: 15 January 2013/Published online: 22 February 2013

(C) The International Gastric Cancer Association and The Japanese Gastric Cancer Association 2013

\begin{abstract}
Background Laparoscopy-assisted total gastrectomy (LATG) for gastric cancer is not yet widespread because of the technical difficulty of reconstruction. We have performed LATG on 100 patients with clinical stage I gastric cancer. This study investigated the short-term outcomes of LATG.

Methods Between September 2001 and September 2012, 100 patients with clinical stage I gastric cancer underwent LATG with D1 plus beta or D2 lymphadenectomy. Roux-en-Y esophagojejunostomy was performed intracorporeally using end-to-side anastomosis with a circular stapler (the purse-string suture method). The primary endpoint was the proportion of postoperative complications during hospitalization.

Results Mean operation time was 249 min; mean blood loss was $182 \mathrm{ml}$. There were no conversions to open surgery. According to the Clavien-Dindo classification, there were 8 grade II $(8 \%)$ and 10 grade IIIa/b $(10 \%)$ complications. There were no treatment-related deaths or grade IV complications. The most frequent complication was anastomotic or stump leakage (6\%), followed by pancreatic fistula (5\%). Reoperations were required in two patients with leakage.

Conclusions The short-term outcomes of LATG in our study involving 100 patients were outlined. LATG for gastric cancer patients should be attempted preferably in a clinical trial setting by surgeons with sufficient experience in laparoscopic gastrectomy.
\end{abstract}

N. Wada · Y. Kurokawa ( $₫) \cdot$ S. Takiguchi · T. Takahashi · M. Yamasaki - H. Miyata - K. Nakajima - M. Mori - Y. Doki Department of Gastroenterological Surgery, Osaka University Graduate School of Medicine, Osaka, Japan e-mail: ykurokawa@gesurg.med.osaka-u.ac.jp
Keywords LATG $\cdot$ LTG $\cdot$ Laparoscopic · Purse-string suture

\section{Introduction}

The feasibility of laparoscopy-assisted distal gastrectomy (LADG) has been assessed in many studies [1-3]. A multicenter phase II study has demonstrated that LADG can be performed safely by surgeons with sufficient experience [4]. Large-scale phase III trials of LADG versus open distal gastrectomy for clinical stage I gastric cancer are now ongoing in Japan and Korea. Although long-term outcomes have not yet been evaluated, LADG has recently become a common surgical procedure in both countries.

Nevertheless, laparoscopy-assisted total gastrectomy (LATG) is not yet widespread. The reconstruction required in LATG is technically much more difficult than that in LADG. Only a few Korean studies have evaluated the feasibility of LATG with more than 100 patients $[5,6]$, so the safety of LATG is still controversial. We have performed LATG with the purse-string suture anastomosis on 100 patients with clinical stage I gastric cancer. This is the first Japanese study involving 100 patients to evaluate short-term outcomes after LATG.

\section{Methods}

Patients

Between September 2001 and September 2012, 110 consecutive patients with clinical stage I (T1N0M0, T1N1M0, 
and T2NOM0) gastric cancer underwent LATG at the Osaka University Hospital. Cases of stump carcinoma were excluded from this study. Because 10 of 110 cases underwent the OrVil method or the overlap method (side-to-side anastomosis with a linear stapler) anastomosis, we analyzed only 100 cases with the purse-string suture anastomosis in this study. Clinical evaluation of tumor depth (cT) and lymph node metastasis (cN) were determined by preoperative evaluations with both endoscopy and computed tomography. The details of the methods for preoperative $\mathrm{T}$ staging have been reported elsewhere [7]. All tumors were histologically diagnosed as adenocarcinoma of the stomach. Clinical stage was classified according to the Japanese Classification of Gastric Carcinoma, second English edition [8]. Informed consent for LATG was obtained from all patients before surgery.

\section{Surgery}

Surgeons performed LATG and lymph node dissection according to the Japanese Gastric Cancer Treatment Guidelines in principle [9]. Patients with cT1 carcinoma underwent D1 plus beta dissection, including station nos. 7, 8a, and 9. Patients with cT2 disease underwent D2 or D2 minus splenic hilum node (station no. 10). D2 minus station no. 10 was treated as D1 plus beta in this study.

For reconstruction, Roux-en-Y esophagojejunostomy was performed with the purse-string suture method as previously reported [10]. In brief, the esophageal stump was sewn over with interrupted sutures laparoscopically or by using a device called the Endostich, and the anvil of a circular stapler was inserted into the esophageal stump. The purse-string suture was tied and reinforced with a monofilament pre-tied loop. A circular stapler inserted into the distal side of the jejunum was introduced into the abdominal cavity through the minilaparotomy site, and esophagojejunostomy was performed. Anastomotic leaks were evaluated using air insufflation.

All operations were performed or supervised by surgeons with sufficient experience with laparoscopic gastrectomies and who were certified by the Japan Society for Endoscopic Surgery.

\section{Statistical analysis}

The primary endpoint of this study was the incidence of postoperative complications during hospitalization. The grading of complications was based on the Clavien-Dindo classification system [11]. All statistical analyses were performed using SPSS Statistics software, version 20 (Chicago, IL, USA).

\section{Results}

The background characteristics of the 100 patients in this study are shown in Table 1 . Ninety percent of patients were diagnosed as cT1. Only 6 patients $(6 \%)$ had clinically positive lymph nodes.

Surgical results are shown in Table 2. D1 plus beta dissection was performed in 95 cases $(95 \%)$ and D2 in 5 cases $(5 \%)$. All patients received Roux-en-Y reconstruction. Mean operation time was 249 min and mean blood loss was $182 \mathrm{ml}$. No patients required conversion to open surgery. Two of 7 patients who received splenectomy did so because of either preoperative comorbidity of thrombocytopenia or intraoperative bleeding from the splenic vein.

Table 3 lists the postoperative complications that occurred during hospitalization. Clavien-Dindo grade II complications occurred in eight patients $(8 \%)$ whereas those of grade IIIa/b occurred in ten patients $(10 \%)$. There were no treatment-related deaths or grade IV complications. The most frequent complication was anastomotic or stump leakage (6\%), followed by pancreatic fistula (5\%). Among the six leakage cases, four occurred in the esophagojejunal anastomosis, one in the duodenum stump, and one in the duodenum stump and the distal side of the jejunum stump. No patients suffered from anastomotic stricture. Reoperations were required in two patients with leakage.

Table 1 Clinical characteristics

\begin{tabular}{ll}
\hline & $n=100$ \\
\hline Age (years) & 63 \\
Median & $29-85$ \\
Range & \\
Gender & $75(75 \%)$ \\
Male & $25(25 \%)$ \\
Female & \\
Body mass index $\left(\mathrm{kg} / \mathrm{m}^{2}\right)$ & 22.5 \\
Median & $16.2-28.0$ \\
Range & \\
Clinical T & $90(90 \%)$ \\
T1 & $10(10 \%)$ \\
T2 & \\
Clinical N & $94(94 \%)$ \\
N0 & $6(6 \%)$ \\
N1 & \\
Clinical stage & $84(84 \%)$ \\
IA & $16(16 \%)$ \\
IB &
\end{tabular}

Clinical TNM stages were classified according to the Japanese Classification of Gastric Carcinoma, second English edition 
Table 2 Surgical results

\begin{tabular}{ll}
\hline & $n=100$ \\
\hline Lymph node dissection & \\
D1 plus beta ${ }^{a}$ & $95(95 \%)$ \\
D2 & $5(5 \%)$ \\
Combined resection & \\
Spleen & $7(7 \%)$ \\
Gallbladder & $6(6 \%)$ \\
Operation time (min) & \\
Mean \pm SD & $249 \pm 47$ \\
Blood loss (ml) & $182 \pm 183$ \\
Mean \pm SD & \\
Number of dissected lymph nodes & $38 \pm 16$ \\
Mean \pm SD & \\
\hline
\end{tabular}

${ }^{a}$ D2 minus station no. 10 was treated as D1 plus beta in this study

Table 3 Postoperative complications

\begin{tabular}{ll}
\hline & $n=100$ \\
\hline Any complications & $8(8 \%)$ \\
Grade II & $10(10 \%)$ \\
Grade IIIa/b & \\
Leakage & 0 \\
Grade II & $6(6 \%)$ \\
Grade IIIa/b & \\
Pancreatic fistula & $1(1 \%)$ \\
Grade II & $5(5 \%)$ \\
Grade IIIa/b & \\
Bleeding & $4(4 \%)$ \\
Grade II & 0 \\
Grade IIIa/b & \\
Pneumonia & $4(4 \%)$ \\
Grade II & 0 \\
Grade IIIa/b & \\
Bowel obstruction & $2(2 \%)$ \\
Grade II & 0 \\
Grade IIIa/b & $2(2 \%)$ \\
Reoperation & \\
\hline Grading of & \\
\hline
\end{tabular}

Grading of complications was based on the Clavien-Dindo classification

\section{Discussion}

Laparoscopy-assisted total gastrectomy is still not widespread because of the technical difficulty of the reconstruction. Several reports have been issued on the feasibility of LATG, but only a few Korean studies have evaluated the feasibility of LATG in populations of more than 100 patients $[5,6]$. We have performed LATG with the purse-string suture anastomosis in 100 patients with clinical stage I gastric cancer. Compared with the previous Korean studies and a small-scale Japanese study evaluating the safety of LATG $[5,6,12,13]$, we were able to perform LATG with more favorable surgical results in terms of operation time. Regarding the incidence of postoperative complications, our study showed better or similar results compared to previous studies of LATG. The incidence of anastomotic leakage in our study was $6 \%(6 / 100)$, including 1 case of duodenum stump leakage and 1 case of leakage of the duodenum stump and the distal side of the jejunum stump. Previous randomized controlled studies have reported that incidence rates of anastomotic leakage after open total gastrectomy ranged from $3.8 \%$ to $6.8 \%$ [14-16]. Nomura et al. [17] reported the result of a retrospective large-scale study of open total gastrectomy. Although they reported the esophagojejunal leakage rate after stapled anastomosis as $1.0 \%$ using only the data of the recent 6 years, the overall incidence of esophagojejunal anastomosis leakage was $2.9 \%$ (27/943). Indeed, our result of the incidence of esophagojejunal anastomosis leakage $(4.0 \%)$ was slightly higher than their result, so we think we should continue to make efforts for reducing the complication rate.

In this study we used only the purse-string suture anastomosis method. The purse-string suture method is simple and is similar to the anastomosis method in open total gastrectomy. The safety of this method has been already reported by other institutions [18, 19]. It requires fewer devices and costs less, and has the advantage of only rarely causing stenosis. Besides the purse-string suture method, two anastomosis methods (the OrVil method and the overlap method) have been reported as useful anastomosis procedures in LATG [20-23]. Although we have performed LATG with the OrVil method or the overlap method for ten cases outside this study, the incidence of anastomotic leakage of grade IIIa/b was $30 \%$ (3/10). The reason for this high incidence was considered to be the inexperience of the surgeons with these methods. These methods might be safer if they were performed more frequently, thus increasing our overall level of expertise.

The risk of postoperative complications is affected by the skill of each individual surgeon. In our case series, six surgeons performed LATG. There was no clear difference among them in the incidence of postoperative complications. Furthermore, all operations were performed or supervised by surgeons with sufficient experience with laparoscopic gastrectomy and who were certified by the Japan Society for Endoscopic Surgery. Also, all surgeons had abundant experience with open gastrectomy. With regard to the learning curve, the incidence of postoperative complications did not show a clear decrease despite the surgeons' increasing expertise. However, in the recent 2 years (after January 2011), there was only one pancreatic 
fistula and no anastomotic leakage. During this period, a fixed team of two surgeons (S.T. and Y.K.) have performed LATG in most cases. Even if the learning curves of individual surgeons do not affect the incidence of complications, a fixed team consisting of the same surgeons could perform LATG more safely.

At this point there are insufficient data concerning longterm outcomes after LATG. Several ongoing randomized control trials are comparing long-term survival between laparoscopic and open distal gastrectomy. Long-term outcomes after LATG should be also evaluated by randomized control trials to establish the possibility of a new standard for the surgical treatment of clinical stage I gastric cancer.

In conclusion, the short-term outcomes of LATG in our study involving 100 patients have been outlined. LATG for gastric cancer patients should be attempted preferably in the clinical trial setting by surgeons with sufficient experience in laparoscopic gastrectomy.

\section{References}

1. Yoshikawa T, Cho H, Rino Y, Yamamoto Y, Kimura M, Fukunaga $\mathrm{T}$, et al. A prospective feasibility and safety study of laparoscopy-assisted distal gastrectomy for clinical stage I gastric cancer initiated by surgeons with much experience of open gastrectomy and laparoscopic surgery. Gastric Cancer 2012; [Epub ahead of print].

2. Zeng YK, Yang ZL, Peng JS, Lin HS, Cai L. Laparoscopyassisted versus open distal gastrectomy for early gastric cancer: evidence from randomized and nonrandomized clinical trials. Ann Surg. 2012;256:39-52.

3. Vinuela EF, Gonen M, Brennan MF, Coit DG, Strong VE. Laparoscopic versus open distal gastrectomy for gastric cancer: a meta-analysis of randomized controlled trials and high-quality nonrandomized studies. Ann Surg. 2012;255:446-56.

4. Katai H, Sasako M, Fukuda H, Nakamura K, Hiki N, Saka M, et al. Safety and feasibility of laparoscopy-assisted distal gastrectomy with suprapancreatic nodal dissection for clinical stage I gastric cancer: a multicenter phase II trial (JCOG0703). Gastric Cancer. 2010;13:238-44.

5. Jeong GA, Cho GS, Kim HH, Lee HJ, Ryu SW, Song KY. Laparoscopy-assisted total gastrectomy for gastric cancer: a multicenter retrospective analysis. Surgery (St. Louis). 2009;146:469-74.

6. Jeong O, Ryu SY, Zhao XF, Jung MR, Kim KY, Park YK. Shortterm surgical outcomes and operative risks of laparoscopic total gastrectomy (LTG) for gastric carcinoma: experience at a largevolume center. Surg Endosc 2012; [Epub ahead of print].

7. Makino T, Fujiwara Y, Takiguchi S, Tsuboyama T, Kim T, Nushijima $\mathrm{Y}$, et al. Preoperative $\mathrm{T}$ staging of gastric cancer by multi-detector row computed tomography. Surgery (St. Louis). 2011;149:672-9.

8. Japanese Gastric Cancer Association. Japanese classification of gastric carcinoma, 2nd English edition. Gastric Cancer 1998;1: $10-24$.
9. Japanese Gastric Cancer Association. Gastric cancer treatment guidelines. Tokyo: Kanehara; 2004. (in Japanese).

10. Takiguchi S, Sekimoto M, Fujiwara Y, Miyata H, Yasuda T, Doki $\mathrm{Y}$, et al. A simple technique for performing laparoscopic purse-string suturing during circular stapling anastomosis. Surg Today. 2005;35:896-9.

11. Dindo D, Demartines N, Clavien PA. Classification of surgical complications: a new proposal with evaluation in a cohort of 6336 patients and results of a survey. Ann Surg. 2004;240: 205-13.

12. Eom BW, Kim YW, Lee SE, Ryu KW, Lee JH, Yoon HM, et al. Survival and surgical outcomes after laparoscopy-assisted total gastrectomy for gastric cancer: case-control study. Surg Endosc 2012; [Epub ahead of print].

13. Sakuramoto S, Kikuchi S, Futawatari N, Katada N, Moriya H, Hirai K, et al. Laparoscopy-assisted pancreas- and spleen-preserving total gastrectomy for gastric cancer as compared with open total gastrectomy. Surg Endosc. 2009;23:2416-23.

14. Schardey HM, Joosten U, Finke U, Staubach KH, Schauer R, Heiss A, et al. The prevention of anastomotic leakage after total gastrectomy with local decontamination. A prospective, randomized, double-blind, placebo-controlled multicenter trial. Ann Surg. 1997;225:172-80.

15. Bozzetti F, Marubini E, Bonfanti G, Miceli R, Piano C, Crose N, et al. Total versus subtotal gastrectomy: surgical morbidity and mortality rates in a multicenter Italian randomized trial. The Italian Gastrointestinal Tumor Study Group. Ann Surg. 1997;226: 613-20.

16. Sano T, Sasako M, Shibata M, Yamamoto S, Tsuburaya A, Nashimoto A, et al. Randomized controlled trial to evaluate splenectomy in total gastrectomy for proximal gastric carcinoma (JCOG0110): analyses of operative morbidity, operation time, and blood loss. J Clin Oncol 2010;28(suppl):15 (abstr 4020).

17. Nomura S, Sasako M, Katai H, Sano T, Maruyama K. Decreasing complication rates with stapled esophagojejunostomy following a learning curve. Gastric Cancer. 2000;3:97-101.

18. Kinoshita T, Oshiro T, Ito K, Shibasaki H, Okazumi S, Katoh R. Intracorporeal circular-stapled esophagojejunostomy using handsewn purse-string suture after laparoscopic total gastrectomy. Surg Endosc. 2010;24:2908-12.

19. So KO, Park JM. Totally laparoscopic total gastrectomy using intracorporeally hand-sewn esophagojejunostomy. J Gastric Cancer. 2011;11:206-11.

20. Inaba K, Satoh S, Ishida $Y$, Taniguchi K, Isogaki J, Kanaya S, et al. Overlap method: novel intracorporeal esophagojejunostomy after laparoscopic total gastrectomy. J Am Coll Surg. 2010;211: e25-9.

21. Tsujimoto H, Uyama I, Yaguchi Y, Kumano I, Takahata R, Matsumoto $\mathrm{Y}$, et al. Outcome of overlap anastomosis using a linear stapler after laparoscopic total and proximal gastrectomy. Langenbecks Arch Surg. 2012;397:833-40.

22. Jeong O, Park YK. Intracorporeal circular stapling esophagojejunostomy using the transorally inserted anvil (OrVil) after laparoscopic total gastrectomy. Surg Endosc. 2009;23:2624-30.

23. Omori T, Oyama T, Mizutani S, Tori M, Nakajima K, Akamatsu $\mathrm{H}$, et al. A simple and safe technique for esophagojejunostomy using the hemidouble stapling technique in laparoscopy-assisted total gastrectomy. Am J Surg. 2009;197:e13-7. 